\title{
Detection of Signature Based Forgeries Using Artificial Neural Network
}

\author{
G.Bharatha Sreeja, T.M.Inbamalar, S.Ciyamala Kushbu, A.Lasipa, A.S.Aileen Jocy
}

\begin{abstract}
Signature plays an important role in banking, financial, commercial etc. Signature may be unique for each person. In olden days, no techniques were used to find the forged signature and it becomes a tremendous strain for human brain. Sometimes the forged signature may also believe as an original one. But nowadays, there are so many methods to detect the forged signature. This paper explains about identifying the forged signature from original signature. The signatures are preprocessed then the features such area, centroid coordinate, eccentricity, kurtosis are extracted. Then it is classified using Artificial Neural Network effectively. The result is analyzed by changing the hidden nodes present in the Neural Network. The performance is evaluated using the parameters such as TPR, TNR, FPR and FNR.
\end{abstract}

Keywords: Artificial Neural Network, Preprocessing, Thinning and Dilation, Feature Extraction, TPR (True Positive Rate), TNR (True Negative Rate), FPR (False Positive Rate) and FNR (False Negative Rate).

\section{INTRODUCTION}

The need for signature verification is very much important because unlike passwords signature cannot be changed or forgotten because it is unique for everyone and it is considered as the important method for verification [1]. The techniques and system used to solve signature verification is divided into offline signature and online signature method [2]. In offline signature verification method more number of hardware's were not used and images were captured using camera, whereas in online verification method more hardware's were used and the hardware's were directly connected to the computer [4]. The features used for offline verification are simpler. The signatures from the database are preprocessed using various preprocessing techniques then the preprocessed database features were extracted. The last stage is that the original and forged signatures were classified using Neural Network [3]. It is a classifier which is used to classify the original and the forged signature. A unique parameter is our signature which is a gained behavioural biometric of a person to show his/her unique identity on documents [11].

Revised Manuscript Received on November 27, 2019.

* Correspondence Author

G. Bharatha Sreeja, RMK College of Engineering and Technology,

Thiruvallur district, Tamilnadu, India

Email: bharatha sreejaece@rmkcet.ac.in

Dr.T.M.Inbamalar, RMK College of Engineering and Technology, Thiruvallur district, Tamilnadu, India

Ciyamala Kushbu, RMK College of Engineering and Technology,

Thiruvallur district, Tamilnadu, India.

A.Lasipa, PET Engineering College, Tamilnadu, India.

A.S.Aileen Jocy. PET Engineering College, Tamilnadu, India.

\section{ARTIFICIAL NEURAL NETWORKS}

Artificial Neural Network has started as a specialized field in engineering disciples. This focuses on pattern verification, and also on development of systems. Neural Networks are computing systems which behaves like a human brain. It has highly interconnected processing elements, these elements helps to identify the forged one.

A Perceptron is the earliest Neural Network model developed by Rosenblatt in 1962. In this model neuron takes weighted sum of inputs and sends the output if it is greater than threshold value. The inputs are $F_{1}, F_{2} \ldots F_{n}$ and weights are $\mathrm{W} 1, \mathrm{~W}_{2} \ldots \ldots . . . \mathrm{W}_{\mathrm{n}}$. When comparing Neural Networks with conventional computers this uses different approaches. A Neural Network can be easy to use, it is an self organization [10].

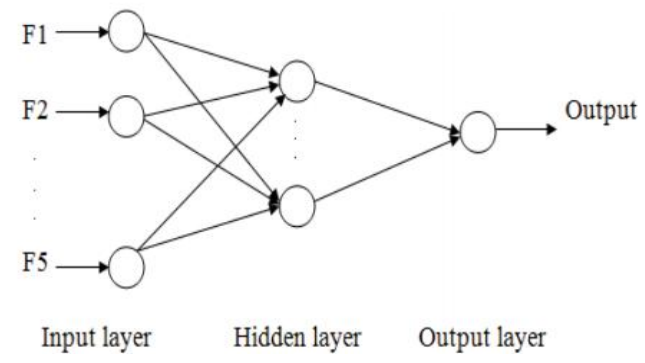

Fig.1. Structure of Artificial Neural Network

\section{A. Algorithm}

Step1: Database from various persons were collected. Step 2: Preprocessing the signatures.

Step 3: Features were extracted for the preprocessed image.

Step 4: Training the Neural Network. Then by changing the hidden nodes performance is noted.

\section{PREPROCESSING}

Preprocessing is the process of improving the image quality and by suppressing unwanted distortion or enhances the image features for further processing. Some of the preprocessing steps performed are given below 


\section{Detection of Signature Based Forgeries Using Artificial Neural Network}

\section{A. Thinning}

Thinning is one of the preprocessing used to remove selected pixels from a binary image.

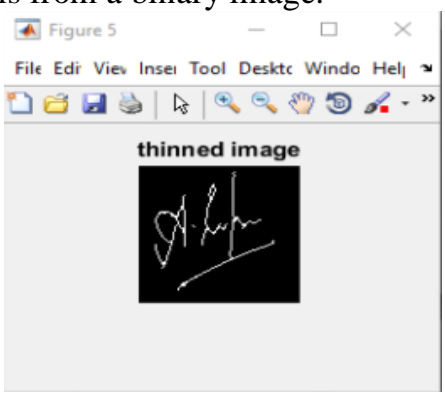

Fig. 2. Thinned image

\section{B. Dilation}

Dilation is the preprocessing method in which pixels are added to the boundary of image

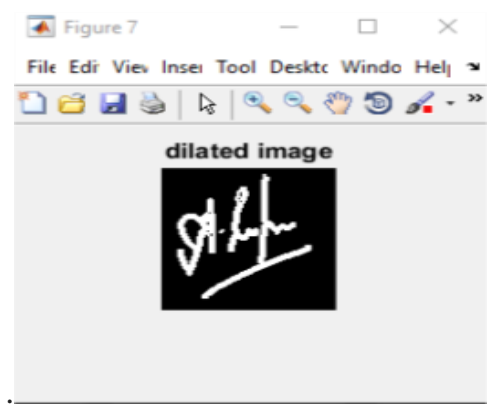

Fig. 3. Dilated image

\section{Noise Removal}

There are various noises in digital image. This noise results in damaging further process. Hence noise removal is applied

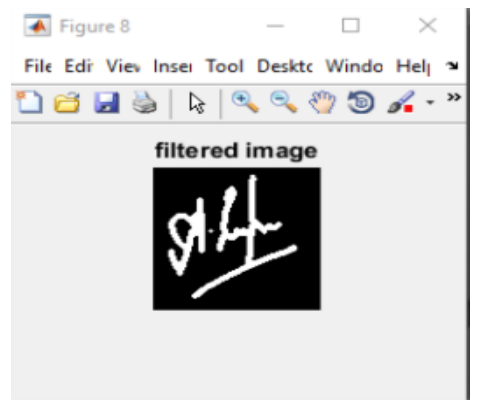

Fig. 4. Filtered image

\section{FEATURE EXTRACTION}

Some of the features like area, centroid coordinate, kurtosis and skewness are extracted from image for further classification. The sample extracted features from signatures are given in the table 1 .

\begin{tabular}{|c|c|c|c|c|c|}
\hline Sign & Area & \multicolumn{2}{|c|}{$\begin{array}{c}\text { Centroid } \\
\text { coordinate }\end{array}$} & $\begin{array}{c}\text { Kurtos } \\
\text { is }\end{array}$ & Skewness \\
\hline 1 & 29.54 & 7.0596 & 6.0725 & 5.0893 & 2.0222 \\
\hline 2 & 82.21 & 11.245 & 10.5826 & 4.2397 & 1.7999 \\
\hline 3 & 85.89 & 7.5118 & 7.2875 & 7.3673 & 2.3673 \\
\hline 4 & 83.07 & 8.1067 & 9.5891 & 7.1307 & 2.0324 \\
\hline
\end{tabular}

Table- 1: Sample extracted features from Signatures

\section{A. Area}

The area is a scalar value which correspond to the total pixel of the image

\section{B. Centriod Co-ordinate}

Centroid coordinate is the center of image. Here both the $\mathrm{X}$ and $\mathrm{Y}$ coordinate of the image are determined.

\section{Kurtosis}

It is measure which determines whether the image is heavy tailed or lightly tailed.

\section{Skewness}

It is the measure of asymmetry of probability of distribution.

\section{TRAINING}

The flow chart for signature verification is shown below

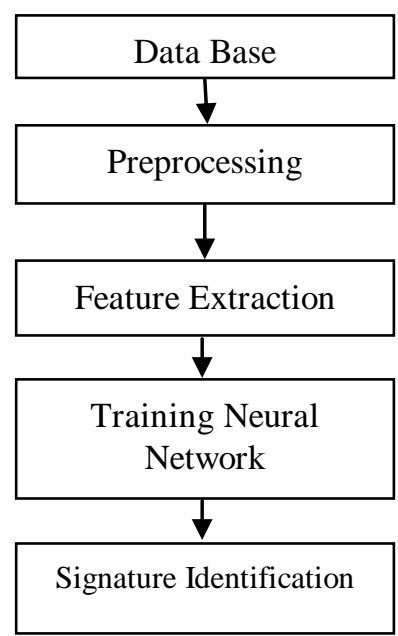

Fig. 5. Flow chart for forgery detection

First the input signatures are collected from various persons and they are stored then the databases were preprocessed [5].Then the features were extracted for the preprocessed image then the neural network is trained to classify whether the signature is original or forged. The Neural Network needs to learn the relationship between the original and forged signature. Once it has been learned the relationship between the original and forged is correctly detected [6]. For classification and verification Back Propagation approach is used. Back propagation approach is the standard one of all other Neural Networks [7]. Back propagation usually has three layers input layer, output layer and hidden layer [8].

Input layer in which the inputs were given. Hidden layer lies between the input and the output layer, and this layer is used to transmit the data from first to second node. Output layer holds the output from the hidden layer 


\section{VERIFICATION}

For the given database the original and the forged signatures were found. The true positive, true negative, false positive, false negative. Values were also found [9].

\section{A. True Positive}

This defines that the Neural Network has correctly classified the original signature.

It is also known as sensitivity.

$$
\mathrm{TRP}=\mathrm{TP} /(\mathrm{TP}+\mathrm{FN})
$$

\section{B. True Negative}

This defines that the Neural Network has correctly classified the Forged signature.

It is also known as specificity

$$
(\mathrm{TNR})=\mathrm{TN} /(\mathrm{FP}+\mathrm{TN})
$$

\section{False Positive}

This defines that the Neural Network has identified the forged signature as original signature.

\section{False Negative}

This defines that the Neural Network has identifies the original signature as forged Signature.

\section{E. Accuracy}

Accuracy is the percentage of correctly classified instances

Accuracy $=(\mathrm{TP}+\mathrm{TN}) / \mathrm{N}$

\section{RESULTS AND DISCUSSION:}

The Neural Network when presented with 18 signature i.e) 9 forged and 9 original.The Neural Network is trained with different hidden values. For 5 hidden nodes it is correctly classified. And Neural Network provides more accuracy. Correct classification, incorrect classification of signatures in percentage is given below.

Table-2: For various hidden nodes the percentage of correct and incorrect classification

\begin{tabular}{|c|c|c|}
\hline $\begin{array}{c}\text { No. of } \\
\text { Hidden } \\
\text { Nodes }\end{array}$ & $\begin{array}{c}\text { Correct } \\
\text { classification } \\
(\%)\end{array}$ & $\begin{array}{c}\text { Incorrect } \\
\text { classification } \\
(\%)\end{array}$ \\
\hline 5 & 95 & 5 \\
\hline 10 & 25 & 7 \\
\hline 20 & 75 & 5 \\
\hline 50 & 75 & 5 \\
\hline
\end{tabular}

Table-3: For different hidden nodes TPR, TNR, FPR, FNR

in percentage

\begin{tabular}{|c|c|c|c|c|}
\hline $\begin{array}{c}\text { Hden } \\
\text { Nodes }\end{array}$ & $\begin{array}{c}\text { TPR } \\
(\%)\end{array}$ & $\begin{array}{c}\text { TNR } \\
(\%)\end{array}$ & $\begin{array}{c}\text { FPR } \\
(\%)\end{array}$ & $\begin{array}{c}\text { FNR } \\
(\%)\end{array}$ \\
\hline 5 & 95 & 95 & 5 & 5 \\
\hline 10 & 50 & 0 & 50 & 100 \\
\hline 20 & 100 & 50 & 0 & 50 \\
\hline
\end{tabular}

\begin{tabular}{|l|l|l|l|l|}
\hline 30 & 75 & 100 & 25 & 0 \\
\hline 50 & 66.67 & 100 & 33.33 & 0 \\
\hline
\end{tabular}

The TPR, TNR, FPR, FNR found for the signature database for different hidden nodes are given in the above table.

\section{CONCLUSION}

The researchers are trying to create the best signature verification tools using advanced algorithms in image processing. The main aim of this work is to develop an efficient offline handwritten signature verification system, in order to achieve good efficiency. The signature verification is performed for database of 18 signatures. Then the signature is classified using neural network. For different hidden nodes correct and incorrect classifications are measured. When hidden nodes are changed to correct value, the correctly classified efficiency achieved is $95 \%$ and incorrectly classified is $5 \%$. Thus while using 5 hidden nodes $95 \%$ result is obtained.

The future work of the current is an automatic feature extraction tools may be developed to predict the relevant features, which will define each signature and reduce the complexity of verification effort.

\section{REFERENCES}

1. Bradley Schafer, Serestina Viriry “An Offline Signature Verification system” IEEE International conference on signals and image processing application, 2009.

2. R. Plamondon and S.N. Srihari, "Online and Offline Handwriting Rec-ognition: A Comprehensive Survey", IEEE Tran. on Pattern Analysis and Machine Intelligence, vol.22 no.1, pp.63-84, Jan.2000.

3. Rajpal Kaur, Pooja Choudhary, "Handwritten Signature Verification Based on Surf Features Using Hmm", IJCST-Volume 3 Issue 1, Jan-Feb 2015.

4. Ashwini Pansare, Shalini Bhatia, "Off-line Signature Verification Using Neural Network" , International Journal of Scientific \& Engineering Research, Volume 3, Issue 2, February-2012.

5. Anu Rathi, Divya Rathi, Parmanand Astya, " Offline handwritten Signature Verification by using Pixel based Method", International Journal of Engineering Research \& Technology (IJERT), Vol. 1 Issue 7, September - 2012.

6. Pradeep Kumar, Shekhar Singh, Ashwani Garg .Nishant Prabhat," Hand Written Signature Recognition \& Verification using Neural Network" International Journal of Advanced Research in Computer Science and Software Engineering, Volume 3, Issue 3, March 2013.

7. Anwar Yahy Ebrahim, Ghazali Sulong," Offline Handwritten Signature Verification Using Back Propagation Artificial Neural Network Matching Technique" Journal of Theoretical and Applied Information Technology, 31st July 2014. Vol. 65 No.3.

8. Pradeep Kumar, Shekhar Singh, Ashwani Garg .Nishant Prabhat," Hand Written Signature Recognition \& Verification using Neural Network" International Journal of Advanced Research in Computer Science and Software Engineering, Volume 3, Issue 3, March 2013.

9. Kevin Woods, Kevin W. Bowyer," Generating ROC Curves for Artificial Neural Networks" IEEE

Transactions on Medical Imaging, Vol. 16, No. 3, June 1997.

10. Ashwini Pansare, Shalini Bhatia, “ Off-line Signature Verification Using Neural Network" , International Journal of Scientific \& Engineering Research, Volume 3, Issue 2, February-2012.

11. Walid Hussein, Mostafa A. Salama and Osman Ibrahim, "Image Processing Based Signature Verification Technique to Reduce Fraud in Financial Institutions", MATEC Web of Conferences ,2016.

12. Gopichand G, Sailaja G, N. Venkata Vinod Kumar, T. Samatha"Digital Signature Verification Using Artificial Neural Networks", International Journal of Recent Technology and Engineering (IJRTE), Volume-7, Issue-6S, March 2019 


\section{AUTHORS PROFILE}

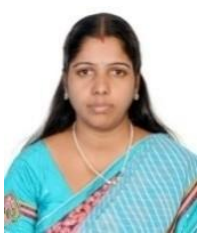

G.Bharatha Sreeja has more than 7 years of teaching experience in TamilNadu and AndhraPradesh. She has published 22 papers in various International Journals and published 14 papers in National and International conference proceedings. She has received Rastrapathi award from former President of India Dr.A.P.J Abdulkalam.

She has received Rajyapurashkar award from Former Governor of TamilNadu. Also she got Best paper award paper for project presentation. She has published 2 books for ECE students. She is the lifetime member of ISTE. Her areas of interest includes Image Processing, Signal Processing and Soft computing.

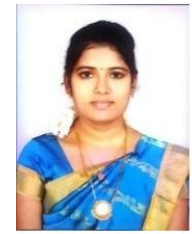

Dr Inbamalar T M has been actively involved in teaching and research for the past 22 years. She has published 32 research papers in various International Journals, National Journals as well as international conferences. She is a reviewer of International Journals such as FEBS Letters, Journal of scientific research and reports, British journal of Applied Science and Technology, Asian Journal of Mathematics and Computer Research, Plant Cell Biotechnology, Molecular Biology, etc. She is editorial board member of International Journal of Emerging Trends in Engineering Research. She is International scientific committee member for various International conferences organized by World Academy of Sciences. She is a Lifetime member of Professional bodies such as ISTE, IACSIT, IAENG and ISRD. She has organized many National Conferences, Faculty development training programs and workshops. She has delivered lectures on various training programs related to research and publications. She has supervised over $50 \mathrm{UG}$ and PG projects. She has been recognized as research supervisor of Anna University since 2016 She has been teaching subjects such as Digital Signal Processing, Advanced Digital Signal Processing, Digital Image Processing, Signals and systems and Medical electronics for UG and PG students.

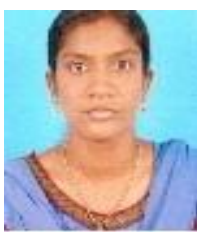

Ciyamala Kushbu has 5 years of teaching experience in Engineering Colleges affiliated to Anna University. She has published 4 papers in International Journals and published 10 papers in various conferences. She has published book titled "Advanced Computer Architecture" topics covered as per syllabus of Anna University regulation 2013. She is also acting as course instructor for CCNA and train many students every year in the field of networking. She is the lifetime member of ISTE. Her areas of interest includes Image Processing, circuit analysis and machine learning.

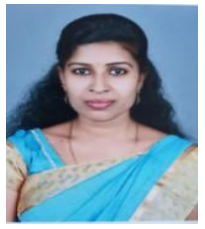

A.Lasipa is a Engineering Graduate and has participated in various project competitions and have won prizes. She has completed various certifications in NPTEL. She is also active member of technical club in college. She has received prizes in project competition. Her area of interests are in Image processing and allied fields.

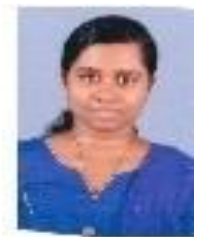

A.S.Aileen Jocy is a Engineering Graduate and has participated in various project competitions and have won prizes. She has completed various certifications in NPTEL. She is also active member of technical club in college. She has received prizes in project competition. Her area of interests are in Soft Computing and allied fields. 